\title{
Measurement and pharmacokinetic analysis of imipramine and its metabolite by brain microdialysis
}

\author{
Yuji Sato, Shinji Shibanoki, Megumi Sugahara \& ${ }^{1}$ Koichi Ishikawa
}

Department of Pharmacology, Nihon University School of Medicine, Oyaguchi-Kami Machi, Itabashi, Tokyo 173, Japan

1 The feasibility of the brain microdialysis method for direct measurement and pharmacokinetic study of imipramine (Imip) and its metabolite desipramine (DMI) was investigated in the rat brain.

2 A dialysis tube was inserted into the right striatum of male Wistar rats, which were administered i.p. with $12.5 \mathrm{mg} \mathrm{kg}^{-1}$ Imip. Thirty $\mu \mathrm{l}$ dialysate was collected every $15 \mathrm{~min}$, and the levels of Imip and DMI were measured by high-performance liquid chromatography with electrochemical detection (h.p.l.c.-e.c.d.). SKF-525A and aminopyrine were concomitantly administered in order to assess their respective effects on the pharmacokinetics of Imip and DMI in the brain.

3 The intracerebral half life $\left(t_{1 / 2}\right)$ of Imip was $2.4 \pm 0.3 \mathrm{~h}$ with Imip alone. Premedication with SKF-525A, an inhibitor of drug-metabolizing enzymes, significantly prolonged the $t_{1 / 2}$ of Imip, while at the same time production of DMI from Imip was accordingly inhibited. Concomitant administration of aminopyrine did not induce any significant change in the concentrations of Imip, but significantly inhibited the concentrations of DMI through its competitive antagonism in the demethylation pathway. 4 The present results suggest that the brain microdialysis method reflects the intracerebral pharmacokinetics of Imip and DMI well and may be applicable to further pharmacokinetic investigations of psychotropic agents.

Keywords: Brain microdialysis; pharmacokinetics; high-performance liquid chromotography with electrochemical detection; imipramine

\section{Introduction}

Imipramine (Imip) is one of the most widely used antidepressants in the clinical practice of psychiatry, and its demethylated metabolite, desipramine (DMI), is also known to exert significant antidepressant activity. While their concentrations in plasma and brain homogenate samples have been often detected by the high performance liquid chromatography (h.p.l.c.) method (Suckow \& Cooper, 1981; Sugita et al., 1987; Foglia et al., 1991), the time course of their concentrations in the brain has rarely been reported, with the exception of an early pharmacokinetic study by Daniel et al. (1981) in which the concentrations of the drugs from animals killed at specific time points were assayed spectrofluorometrically.

Recent advances in brain microdialysis techniques have enabled the direct measurement of various neurotransmitters in the brain (Ungerstedt \& Hallstroem, 1987), but pharmacokinetic investigations of psychotropic agents by this method are limited (Dubey et al., 1989; Matos et al., 1992; Tellez et al., 1992). Since pharmacokinetic drug monitoring is crucial for the rational therapeutic use of drugs, the feasibility of employing the brain microdialysis method for pharmacokinetic studies is of particular importance. One of the methological advantages of brain microdialysis is that, in particular, time-dependent changes in the concentrations of a psychotropic drug administered to a single animal can be easily traced for a length of time that is sufficient to calculate the biological half life and other pharmacokinetic parameters of the drug correctly. This is not achievable by the traditional measurement of drug concentration in the plasma by h.p.l.c., which requires too large an amount of blood to be sampled from one animal.

We established a method for directly monitoring the timecourse of the concentrations of Imip and DMI in the brain of rats by applying the brain microdialysis method and h.p.l.c. with electrochemical detection (e.c.d.). This paper describes the method employed, the pharmacokinetic data

\footnotetext{
' Author for correspondence.
}

obtained for Imip and DMI in brain of rats, and the effects of SKF-525A and aminopyrine of the pharmacokinetics of Imip and DMI. SKF-525A, a well-known inhibitor of liver enzymes involved in drug metabolism (Kato et al., 1964; Galeotti et al., 1983) and aminopyrine, which, together with Imip, is metabolized mainly through demethylation, were administered concomitantly with Imip. The aim was to determine whether or not the time-dependent changes in concentrations of Imip and DMI measured by brain microdialysis correctly reflected the actual pharmacokinetics of Imip and DMI in the rat brain which is influenced by both SKF-525A and aminopyrine.

\section{Methods}

\section{Animals and surgical procedures}

Twenty-one adult male Wistar rats (Shizuoka Laboratory Animal Center, Hamamatsu, Japan), weighing about 300 to $350 \mathrm{~g}$, were used for the experiments. The animals were housed for at least one week prior to the experiments in a room where the temperature $\left(23 \pm 0.5^{\circ} \mathrm{C}\right)$, humidity $(60 \pm 5 \%)$ and light cycle (12-h illumination with the light turned on at $07 \mathrm{~h} 00 \mathrm{~min}$ ) were controlled. The rats were fed on laboratory chow regularly and water ad libitum. They were anaesthetized with a single i.p. injection of $50 \mathrm{mg} \mathrm{kg}^{-1}$ pentobarbitone and positioned in a stereotaxic frame with atraumatic ear bars. After exposing the skull, a guide cannula (G-8, Eicom, Kyoto, Japan) was implanted stereotaxically into the right striatum (coordinates with respect to the bregma: anterior, 0; lateral, 3 ; ventral, $4 \mathrm{~mm}$ ) according to the atlas of Paxinos \& Watson (1982). After implantation, the cannula was fixed firmly to the skull with anchor screws and dental cement, and then occluded with a dummy cannula. The dialysis experiment was performed at least 2 days after the operation in order to avoid possible interfering effects. 


\section{Drug administration}

Imip at a dose of $12.5 \mathrm{mg} \mathrm{kg}^{-1}$ was injected i.p. into all the animals. In order to evaluate the influence of SKF-525A on the elimination of Imip, $50 \mathrm{mg} \mathrm{kg}^{-1}$ SKF-525A was injected i.p. into 5 animals 30 min before the administration of Imip. Aminopyrine was co-administered with Imip to 11 animals at dose levels of 25,50 , and $100 \mathrm{mg} \mathrm{kg}^{-1}$ i.p. (the number of the animals given each dose of aminopyrine were 3,4 and 4 , respectively) to determine how it affected the demethylation of Imip and the production of DMI. All the drugs were given in a volume of $0.1 \mathrm{ml} 100 \mathrm{~g}^{-1} 2 \mathrm{~h}$ after dialysis started.

\section{Dialysis}

On the day of the experiment, the dummy cannula was removed from the guide cannula and an I-shaped dialysis probe (BDP-I-8-03, Eicom; mol. wt. cutoff :50000; exposed surface for striatum $: 3.0 \mathrm{~mm}$ ). was inserted which was then perfused with Ringer solution (composition mM: $\mathrm{NaCl}$ 147, $\mathrm{KCl} 4.0, \mathrm{CaCl}_{2} 2.4$ ) at a constant flow rate of $2 \mu \mathrm{min}^{-1}$ using a perfusion pump (Microliter syringe pump, Harvard, U.S.A.). Following a 2-h period for stabilization of the baseline, dialysate samples $(30 \mu \mathrm{l})$ were collected directly into an autoinjector every $15 \mathrm{~min}$ throughout the whole experiment. All rats were placed in a plexiglass container $(30 \times 30 \times 35 \mathrm{~cm})$ during the experiment, kept freely moving and allowed free access to food and water.

The in vitro recovery rates of Imip and DMI were estimated by placing probes in Ringer solution containing Imip and DMI. Collection of the perfusate and assay of the Imip and DMI contained in the perfusate were undertaken in the same manner as described above. The recovery rates of Imip and DMI so calculated were $15.1 \pm 2.1 \%$ and $15.0 \pm 2.2 \%$, respectively (mean \pm s.d., $n=4$ ). The following results have not been corrected for recovery for convenience.

\section{Chromatographic conditions}

The concentrations of Imip and DMI in the dialysates were measured by h.p.l.c.-e.c.d. The h.p.l.c.-e.c.d. system consisted of a pump (880-PU, JASKO, Tokyo, Japan) with an autoinjector (AS-10, Eicom, Kyoto, Japan; injector loop size: $100 \mu \mathrm{l})$, an electrochemical detector (CB-100, Eicom, Kyoto, Japan) with a thin-layer carbon graphite electrode (6-GE, Eicom) and a chromatographic column (average particle size, $5 \mu \mathrm{m}$; $150 \times 6.4 \mathrm{~mm}$ i.d.; Puresil C18 ODS column, Millipore, U.S.A.). The mobile phase consisted of a mixture of $0.1 \mathrm{M}$ sodium acetate buffer ( $\mathrm{pH} 4.0)$, acetonitrile and $\mathrm{H}_{2} \mathrm{O}(5: 3: 2)$, and the flow rate was set at $1.5 \mathrm{ml} \mathrm{min}^{-1}$. The chromatographic column was maintained at $37^{\circ} \mathrm{C}$ with a waterbath (Thermo Minder Mini-80, Taiyo, Tokyo, Japan). The detector potential was set at $800 \mathrm{mV}$ versus an $\mathrm{Ag} / \mathrm{AgCl}$ reference electrode. After the experiment, rats were killed by i.p. injection of thiopentone sodium and the location of each probe was verified histologically.

\section{Drugs and chemicals}

Imipramine hydrochloride was purchased from Nihon CibaGeigy Co. (Hyogo, Japan). Desipramine hydrochloride was purchased from Sigma, St. Louis, U.S.A. Each drug was dissolved in saline immediately prior to injection. Acetonitrile and sodium acetate were obtained from Wako Pure Chemicals, Osaka, Japan. N,N-diethylaminoethyl-2,2diphenylvalerate hydrochloride (SKF-525A hydrochloride) was purchased from Research Biochemicals Inc., U.S.A. Aminopyrine was purchased from Sigma.

\section{Statistics}

Statistical analysis of the data for multiple comparisons was performed by two-way analysis of variance (ANOVA) with repeated measures on one factor and post-hoc tests (Fisher's protected least significance difference; significance level, $5 \%$ ). For single comparisons, the significance of differences between means was determined by Student's two-tailed $t$ tests.

\section{Results}

\section{Static experiments}

Hydrodynamic voltammograms of Imip and DMI are plotted in Figure 1. Both Imip and DMI started their electrical responses at $450 \mathrm{mV}$. The responses increased steadily up to about $850 \mathrm{mV}$ and then decreased. The maximum response was higher in DMI than in Imip.

\section{Chromatograms}

A chromatogram for authentic standard Imip and DMI, a blank chromatogram, and a typical chromatogram obtained from a rat sample after i.p. administration of Imip are illustrated in Figure 2. The retention times of authentic Imip and DMI were $9.80 \mathrm{~min}$ and $8.78 \mathrm{~min}$, respectively. The blank chromatogram did not demonstrate peaks corresponding to those of the authentic standards, while two peaks with the same retention times as those of the authentic standards appeared on the chromatogram from the sample. The present chromatographic conditions thus revealed no significant interference by biological substances in the accurate determination of the drugs, and we can therefore identify the two peaks on the chromatogram from the sample as representing Imip and DMI.

\section{Concentrations of Imip and DMI in dialysate}

The time-course changes in the extracellular concentrations of Imip and DMI in $30 \mu$ l dialysate after a single administration of Imip are shown in Figure 3a. Imip reached a peak at about $60 \mathrm{~min}$ after its administration, while DMI reached its maximal concentration about $180 \mathrm{~min}$ after administration. Notably also, the concentrations of DMI decreased more slowly than those of Imip.

Figure $3 b$ illustrates the time course changes of Imip and DMI following premedication with SKF-525A. The concentrations of DMI at every point were significantly lower $(F(1,39)=17.984, P<0.0001)$ in the group to which SKF$525 \mathrm{~A}$ was administered prior to Imip than in group injected with Imip alone. It is also evident that the concentrations of

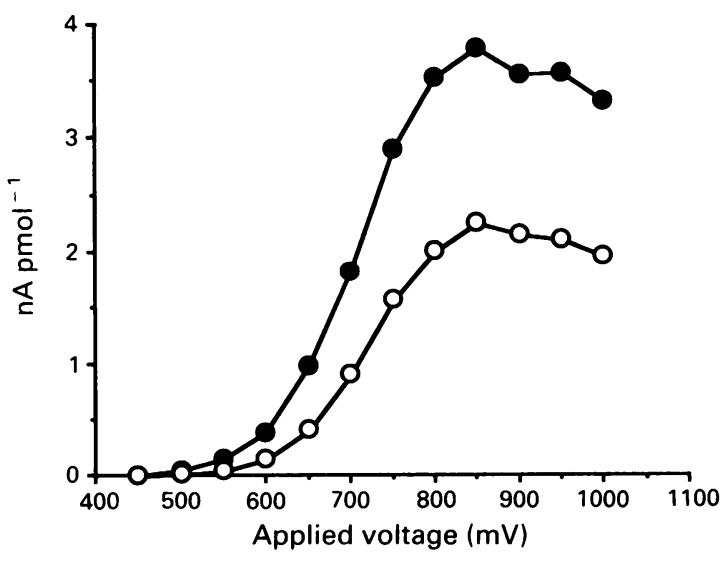

Figure 1 Hydrodynamic voltammograms of imipramine (Imip, O) and desipramine (DMI, O). Detector, CB-100, Eicom; mobile phase, 0.1 M sodium acetate buffer ( $\mathrm{pH} 4.0)$ : acetonitrile: $\mathrm{H}_{2} \mathrm{O}(5: 3: 2)$. Each curve represents $400 \mathrm{ng}$ of Imip and DMI. 


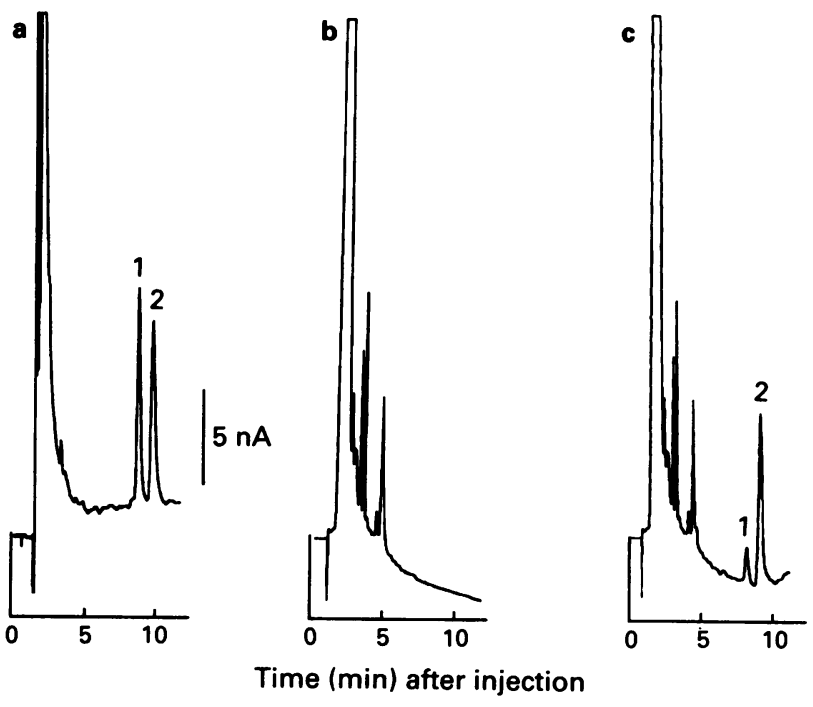

Figure 2 Typical chromatograms of dialysates. (a) Standard substances (400 pg of imipramine (Imip) and desipramine (DMI)); (b) blank; (c) sample after injection of Imip $12.5 \mathrm{mg} \mathrm{kg}^{-1}$. (1) DMI; (2) Imip. The applied voltage of the electrochemical detector was set at $850 \mathrm{mV}$.

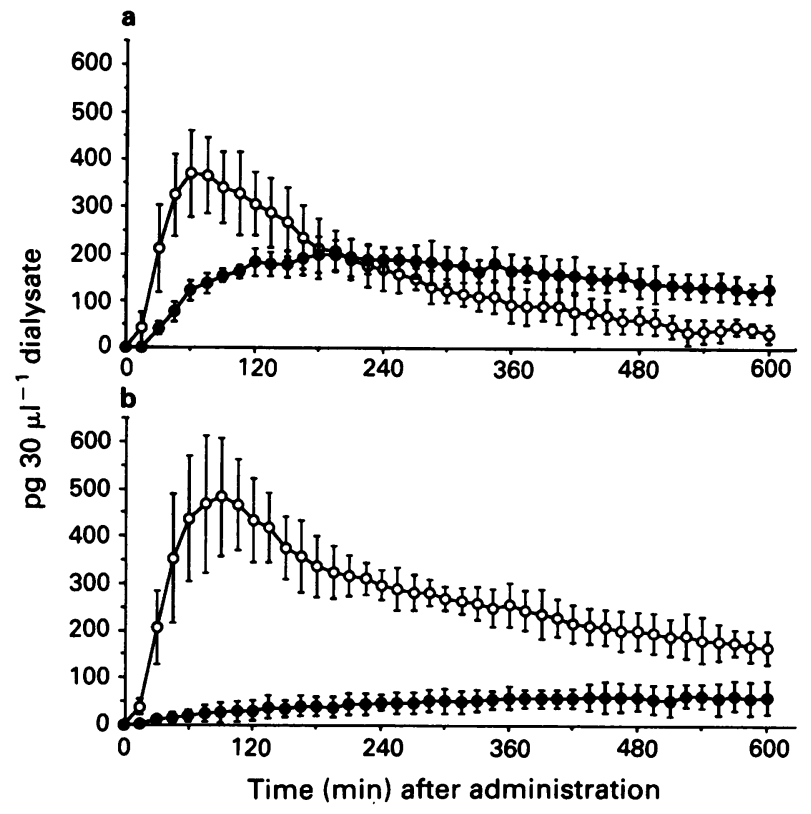

Figure 3 Extracellular concentrations of imipramine (Imip, $O$ ) and desipramine (DMI, ) $\left(\mathrm{pg} 30 \mathrm{\mu l}^{-1}\right.$ dialysate) after intraperitoneal administration of $12.5 \mathrm{mg} \mathrm{kg}-1$ of Imip without $(\mathrm{a} ; n=5)$ and with $50 \mathrm{mg} \mathrm{kg}^{-1}$ of SKF-525A (b, $n=5$ ). Premedication with SKF-525A significantly lowered the concentrations of DMI, while the concentrations of IMP became significantly higher (by ANOVA; $\mathrm{F}(1,39)=17.984, P<0.0001)$.

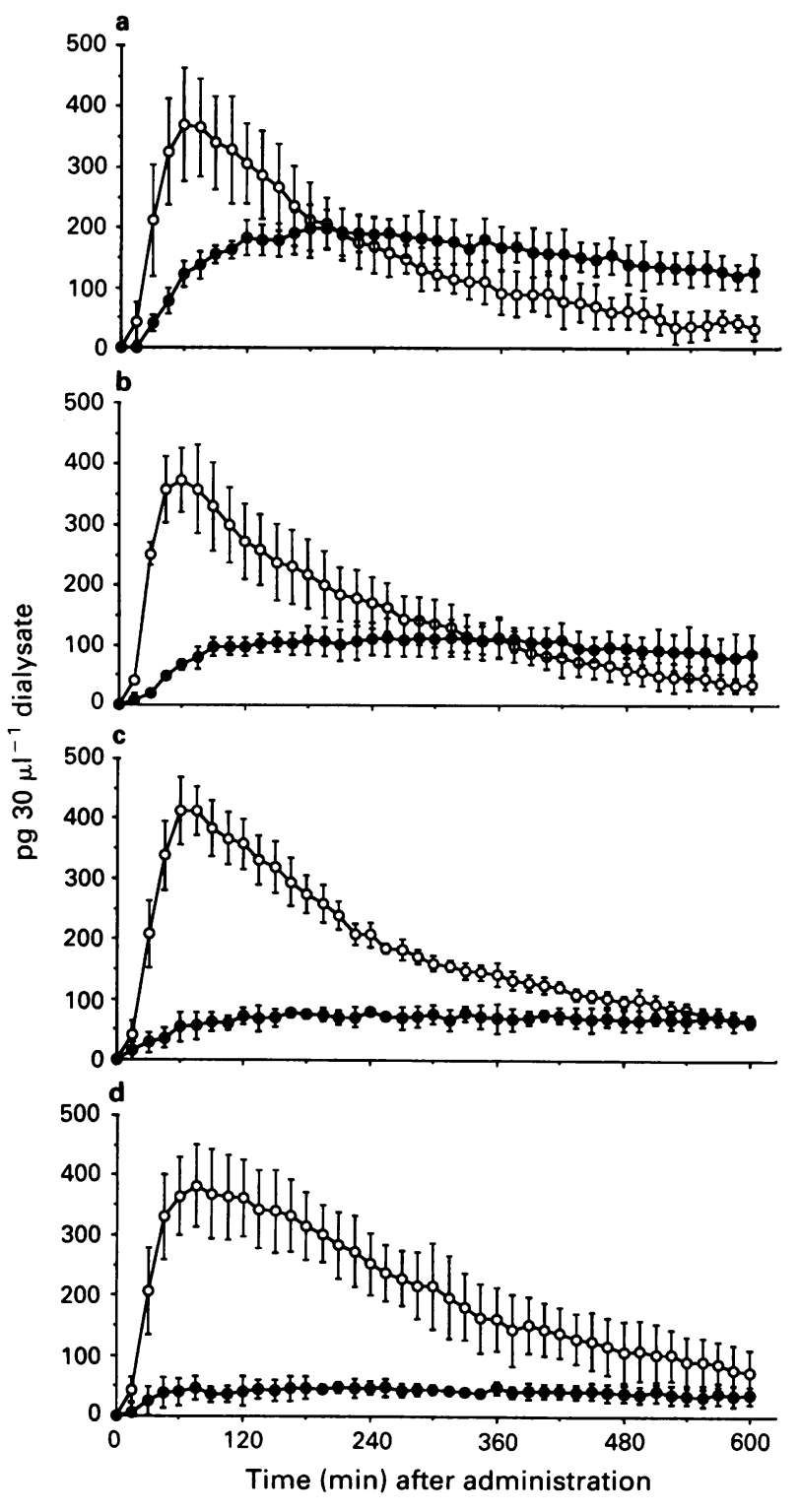

Figure 4 Time-course of imipramine (Imip, O) and desipramine (DMI, ) with concomitant administration of aminopyrine (AMP). (a) ( $n=5)$, Imip only; (b) $(n=3)$, Imip $+25 \mathrm{mg} \mathrm{kg}^{-1}$ AMP; (c) $(n=4)$, Imip $+50 \mathrm{mg} \mathrm{kg}^{-1}$ AMP; (d) $(n=4)$, Imip $+100 \mathrm{mg} \mathrm{kg}^{-1}$ AMP. The extracellular concentrations of DMI showed significant differences in proportion to the given dosage of AMP (by ANOVA; $F(3,177)=3.337, P<0.0001)$, while the concentrations of Imip were not significantly different with or without concomitant administration of AMP (by ANOVA; $F(3,117)=0.969, P>0.01$ ).

Table 1 Biological half life and other pharmacokinetic parameters of imipramine (Imip)

\begin{tabular}{|c|c|c|c|c|}
\hline Treatment & $\mathrm{C}_{o}\left(\mathrm{pg} 30 \mu \mathrm{l}^{-1}\right)$ & $C_{\max }\left(\operatorname{pg} 30 \mu 1^{-1}\right)$ & $\mathrm{t}_{1 / 2}(\mathrm{~h})$ & $\mathbf{k}_{e}\left(\mathrm{~h}^{-1}\right)$ \\
\hline & & 369 & $2.4 \pm 0$ & 0.29 \\
\hline Imip & 479.6 & $441.1 \pm$ & $6.7 \pm 1.4$ & $0.11 \pm 0.0026$ \\
\hline Imip + 25AMP & $516.8 \pm 196.6$ & $373.8 \pm 53.1$ & $2.7 \pm 0.6(1.9-3.0)$ & $0.27 \pm 0.073$ \\
\hline Imip + 50AMP & $559.5 \pm 81.7$ & $412.1 \pm 40.3$ & $3.5 \pm 0.2(3.2-3.6)^{*}$ & $0.19 \pm 0.014$ \\
\hline Imip + 100AMP & $494.5 \pm 54.9$ & $382.2 \pm 68.4$ & $3.5 \pm 0.7(2.6-4.4)^{* *}$ & $0.21 \pm 0.043$ \\
\hline
\end{tabular}

Biological half life $\left(t_{1 / 2}\right)$, elimination rate constant $\left(k_{\mathrm{e}}\right)$ and initial concentration $\left(C_{\mathrm{o}}\right)$ are theoretical values calculated from regression coefficients given by applying the least square method to the log/linear concentrations/time curves of Imip of each rat. $C_{\max }$ represents the mean maximal concentrations of Imip, derived from the linear/linear plots of concentrations of Imip. Significant differences in $t_{1 / 2}$ were observed between the Imip alone group and the Imip + SKF-525A, Imip + $50 \mathrm{mg}$ and $100 \mathrm{mg}$ aminopyrine (AMP) groups, respectively $\left({ }^{*} P<0.01 ;{ }^{* *} P<0.05\right)$. 
Imip in the SKF-525A group were significantly higher than those in the group to which Imip alone was administered $(F(1,39)=42.548, P<0.0001)$. This difference in concentrations of Imip and DMI is considered to be attributable to the effect of SKF-525A, which inhibits the drug metabolism by liver enzymes, such as cytochrome P450, by suppressing the production of DMI from Imip and prolonging the elimination of Imip.

Figure 4 shows the time-course changes in the extracellular concentrations of Imip and DMI with concomitant administration of aminopyrine at dose levels of 25,50 , and $100 \mathrm{mg}$ $\mathrm{kg}^{-1}$. Significant differences $(F(3,117)=3.337, P<0.0001)$ were noted among the extracellular concentrations of DMI in proportion to the given dosages of aminopyrine. These differences are thought to reflect the competitive antagonism between Imip and aminopyrine, both of which are metabolized mainly through demethylation. The fact that the concentrations of Imip with and without concomitant administration of aminopyrine were not significantly different $(F(3,117)=0.969, \quad P>0.01) \quad$ implies that aminopyrine affected only the metabolic pathway through demethylation, so leaving other pathways and the metabolism of Imip as a whole intact.

\section{Pharmacokinetic data}

The biological half life $\left(t_{1 / 2}\right)$, maximal $\left(C_{\max }\right)$ as well as initial $\left(C_{\mathrm{o}}\right)$ concentrations and elimination rate constant $\left(k_{\mathrm{e}}\right)$ of Imip are listed in Table $1 . C_{\mathrm{o}}, t_{1 / 2}$ and $k_{\mathrm{e}}$ were calculated from the regression coefficients given by applying the least square method to the $\log /$ linear concentrations/time curves of Imip between $60-600 \mathrm{~min}$ after the administration of Imip in each rat. The correlation coefficients of the regression curves all exceeded 0.98 and were statistically significant $(P<0.001)$. $C_{\max }$ represents the actual mean value derived from the linear/linear plots of concentrations of Imip as shown in Figures 3 and 4.

Significant differences in $t_{1 / 2}$ were observed between the Imip alone group and the Imip $+50 \mathrm{mg}, 100 \mathrm{mg}$ aminopyrine (AMP) and Imip + SKF-525A (SKF) groups, respectively (Student's two-tailed $t$ tests).

The significant prolongation of the $t_{1 / 2}$ of Imip as a result of concomitant administration of SKF-525A or aminopyrine implies that demethylation plays a major role in the metabolism of Imip and that the prolongation of $t_{1 / 2}$ due to inhibition of drug metabolizing enzymes has a clinical significance. Since it has already been reported that the plasma levels of both Imip and DMI are significantly correlated with their clinical effect (Reisby et al., 1977), the prolonged intracerebral concentration of Imip and resultant low production of DMI may bring about some important differences in the clinical pharmacodynamic effects. No significant difference was demonstrated for $C_{\mathrm{o}}$ (the initial concentration of Imip) or $C_{\max }$ (the maximal concentration of Imip), implying that its uptake into the brain was unaffected by the concomitant administration of either SKF-525A or aminopyrine.

\section{Discussion}

The present findings demonstrate that the brain microdialysis technique, while having been applied almost solely to pharmacodynamic investigations of various neurotransmitters, can also be utilized for pharmacokinetic studies in psychopharmacology which have previously been undertaken mainly by means of the determination of psychotropic drugs in homogenized brain samples from animals (Imip by Masada et al., 1986). The application of microdialysis to the pharmacokinetic study of psychotropic agents, although still limited (diazepam by Dubey et al., 1989; phenobarbitone by Tellez et al., 1992), is of great importance, since microdialysis can detect quite small amounts of psychotropic drugs

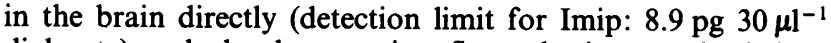
dialysate) and also because it reflects the intracerebral drug metabolism quite well in free-moving rats without gross damage to the brain.

In our experiments, the feasibility and accuracy of the brain microdialysis technique for the pharmacokinetic study of psychotropic agents have been endorsed. First, premedication with SKF-525A prior to the administration of Imip significantly prolonged the elimination of Imip and inhibited the increase in concentration of DMI in the collected dialysates. This means that the concentration of DMI, a demethylated metabolite of Imip, in dialysates from the brain reflected the general drug metabolism through liver enzymes, mainly cytochrome P-450, being inhibited by the administration of SKF-525A. It is well known that there is substantial cytochrome P-450 activity in the brain mitochondria (GhersiEgea \& Livertoux, 1992). Thus, it must be taken into consideration that Imip may be metabolized in part also in the CNS. However, if SKF-525A crosses the blood-brain-barrier in parallel with Imip and affects the cytochrome P-450 localized in the brain, the drug metabolism in the CNS must be inhibited by the systemic administration of SKF-525A to the same extent as the cytochrome P-450 localized in the liver.

Concomitant administration of aminopyrine suppressed the production of DMI from Imip significantly in a dosedependent manner, while it did not affect the time-course changes in concentration of Imip. This is considered to be due to competitive antagonism between aminopyrine and Imip, both of which are metabolized through demethylation. The fact that the elimination of Imip did not change significantly is due to the availability of alternative metabolic pathways other than demethylation, such as hydroxylation (Potter et al., 1982). We thus found, by the brain microdialysis technique, that the dose-dependent competitive antagonism between Imip and aminopyrine, both administered i.p., was well reflected in the dialysates collected through the microdialysis probe. There are several reports in which the competitive antagonism between aminopyrine and other drugs has been investigated (Renton, 1985; Gellert et al., 1986; Moochhala et al., 1986; Floreani \& Carpenedo, 1990). Similar results were obtained in our present experiments.

In a previous study, in which we measured the concentrations of drugs in homogenized brain samples (Shibanoki $e t$ al., 1989), the biological half lives of Imip and DMI in the rat brain after intravenous injection of Imip were estimated to be $114 \pm 24 \mathrm{~min}$ and $210 \pm 54 \mathrm{~min}$, respectively. Despite the difference in mode of administration of Imip, the difference in biological half life of Imip between our two different methods is relatively small, considering that the Imip measured in dialysis only reflected the concentration of the free compound. This confirms that the brain dialysis method, in comparison with previous detection techniques, holds good for the measurement of psychotropic agents.

Daniel et al. (1981) found some deviation from linearity in the Imip and DMI time curves in the blood and the brain and suggested that the pharmacokinetics of Imip and DMI follows the equations for a multi-compartment system. However, the correlation coefficients of the Imip and DMI time curves in our experiment suggested their linearity, which implies a single compartment model for their pharmacokinetics. In our experiment, concentrations of Imip and DMI were measured from the dialysates collected through the probe in the striatum which can be regarded as a single compartment in the pharmacokinetics of Imip and DMI, whereas Daniel et al. (1981) measured Imip and DMI from homogenized samples of the whole brain which must serve as a multi-compartment system.

In conclusion, brain microdialysis combined with h.p.l.c.e.c.d. represents a sensitive procedure for the direct measurement of the time-course concentrations of psychotropic agents in the brain. It is hoped that through the use of this 
method, the pharmacokinetics of various psychotropic agents and their pharmacodynamic effects on neurotransmitters will be examined further, so that the actual mechanisms and metabolism of psychotropic drugs will be better understood and permit their more rational clinical usage.

\section{References}

DANIEL, W., ADAMUS, A., MELZACKA, M., SZYMURA, J. \& VETULANI, J. (1981). Cerebral pharmacokinetics of imipramine in rats after single and multiple dosages. Naunyn-Schmied. Arch. Pharmacol., 317, 209-213.

DUBEY, R.K., MCALLISTER, C.B., INOUE, M. \& WILKINSON, G.R. (1989). Plasma binding and transport of Diazepam across the blood-brain barrier. J. Clin. Invest., 84, 1155-1159.

FLOREANI, M. \& CARPENEDO, F. (1990). Inhibition of rat liver monooxygenase activities by 2-methyl-1,4-naphthoquinone (menadione). Toxicol. Appl. Pharmacol., 105, 333-339.

FOGLIA, J.P., SORISIO, D. \& PEREL, J.M. (1991). Determination of imipramine, despiramine and their hydroxy metabolites by reversed-phase chromatography with ultraviolet and coulometric detection. J. Chromatogr., 572, 247-258.

GALEOTTI, T., EBOLI, M., PALOMBINI, G. VAN ROSSUM, G.D.V. \& KAPOOR, S.C. (1983). Inhibition of mitochondrial oxidative metabolism by SKF-525A in intact cells and isolated mitochondia. Biochem. Pharmacol., 32, 3285-3295.

GELLERT, J., ALDERMAN, J. \& LIEBER, C.S. (1986). Interaction between ethanol metabolism and mixed-function oxidation in alcohol dehydrogenase positive and negative deermice. Biochem. Pharmacol., 35, 1037-1041.

GHERSI-EGEA, J.F. \& LIVERTOUX, M.H. (1992). Evidence for drug metabolism as a source of reactive species in the brain. Exper. Suppl. (Basel), 62, 219-226.

KATO, R., CHIESARA, E. \& VASSANELli, P. (1964). Further studies on the inhibition and stimulation of microsomal drugmetabolizing enzymes of rat liver by various compounds. Biochem. Pharmacol., 13, 69-83.

MASADA, M., SUZUKI, K., KIKUTA, S., YAMASHITA, S., NAKANISHI, K., NADAI, T., IGARASHI, Y. \& NOGUCHI, T. (1986). Regional distribution and elimination kinetics of imipramine in rat brain after a single intraperitoneal administration. Chem. Pharm. Bull., 34, 2173-2177.

MATOS, F.F., ROLLEMA, H. \& BASBAUM, A.I. (1992). Simultaneous measurement of extracellular morphine and serotonin in brain tissue and CSF by microdialysis in awake rats. J. Neurochem., 58, 1773-1781.
MOOCHHALA, S.M. \& RENTON, K.W. (1986). Inhibition of hepatic microsomal drug metabolism by the immunosuppressive agent cyclosporin A. Biochem. Pharmacol., 35, 1499-1503.

PAXINOS, G. \& WATSON, C. (1982). The Rat Brain in Stereotaxic Coordinates, 2nd Edition. New York: Academic Press.

POTTER, W.Z., CALIL, H.M., SUTFIN, T.A., ZAVADIL, A.P., JUSKO, W.J., RAPOPORT, J. \& GOODWIN, F.K. (1982). Active metabolites of impramine and desipramine in man. Clin. Pharmacol. Ther., 31, 393-401.

REISBY, N., GRAM, L., BECH, P., NAGY, A., PETERSEN, G., ORTMANN, J., IBSEN, I., DENCKER, S., JACOBSEN, O., KRAUTWALD, O., SONDERGAARD, I. \& CHRISTIANSEN, J. (1977). Imipramine: clinical effects and pharmacokinetic variability. Psychopharmacology, 54, 263-272.

RENTON, K.W. (1985). Inhibition of hepatic microsomal drug metabolism by the calcium channel blockers diltiazem and verapamil. Biochem. Pharmacol., 34, 2549-2553.

SHIBANOKI, S., IMAMURA, Y., KUBO, T., KOGURE, M., GOTOH, Y. \& ISHIKAWA, K. (1989). Difference in the biological fate of dosulepin and imipramine in rats. Neurosciences, 15, 359-370.

SUCKOW, R.F. \& COOPER, T.B. (1981). Simultaneous determination of imipramine, desipramine, and their 2-hydroxy metabolites in plasma by ion-pair reversed-phase high-performance liquid chromatography with amperometric detection. J. Pharm. Sci., 70, 257-261.

SUGITA, S., KOBAYASHI, A., SUZUKI, S., YOSHIDA, T. \& NAKAZAWA, K. (1987). High-performance liquid chromatographic determination of imipramine and its metabolites in rat brain. J. Chromatogr., 421, 412-417.

TELLEZ, S., FORGES, N., ROUSSIN, A. \& HERNANDEZ, L. (1992). Coupling of microdialysis with capillary electrophoresis: a new approach to the study of drug transfer between two compartments of the body in freely moving mouse. J. Chromatogr., 581, 257-266.

UNGERSTEDT, U. \& HALLSTROEM, A. (1987). In vivo microdialysis: a new approach to the analysis of neurotransmitters in the brain. Life Sci., 41, 861-864. 